\title{
多平台搭配，打造优质在线课
}

\author{
王林艳 ${ }^{1}$, 许国贺 1 , 陈俊英 ${ }^{2}$, 李俊杰 ${ }^{1}$, 马晶军 1 , \\ 1 河北农业大学理工学院, 河北黄骅 061100 \\ 2 河北农业大学理学院, 河北保定 071001
}

\begin{abstract}
摘要: 通过 $\mathrm{QQ}$ 分屏与学习通结合, 叙述了化工原理课程线上授课全过程。详细介绍了课前准备、线上授课模式以及平 台使用技巧, 针对教学中的重点问题以及学生提出的问题给出详细的处理方案。能够帮助零基础的教师快速实现在线教 学, 为广大线上教学教师提供备选方案。
\end{abstract}

关键词: 新型冠状病毒; 线上教学; 学习通; $Q Q$ 分屏

中图分类号: G64; O6

\section{Multi-Platform Collocation to Create Quality Online Courses}

Linyan Wang ${ }^{1}$, Guohe Xu ${ }^{1}$, Junying Chen ${ }^{2}$, Junjie Li ${ }^{1}$, Jingjun Ma ${ }^{1, *}$

${ }^{1}$ College of Science \& Technology, Hebei Agricultural University, Huanghua 061100, Hebei Province, P. R. China.

${ }^{2}$ College of Science, Hebei Agricultural University, Baoding 071001, Hebei Province, P. R. China.

\begin{abstract}
This paper describes the whole process of online teaching of Unit Operations of Chemical Engineering by $\mathrm{QQ}$ split screen and supernovae learning. The pre-class preparation, online teaching mode and using skills are presented in detail. Moreover, the processing schemes are presented to deal with the key problems and students' questions. It can help teachers with zero online teaching experiences implement online teaching, which certainly provides alternative methods for teachers.
\end{abstract}

Key Words: Novel coronavirus; Online teaching; Supernovae learning; QQ split screen

2020 年 1 月, 突如其来的新冠肺炎席卷全国 ${ }^{[1]}$, 全国已确诊和疑似病例人数逐日攀升。出于疫 情防控的需要, 教育部做出推迟开学的决定, 并于 2 月 4 日发布了《关于在疫情防控期间做好普通 高等学校在线教学组织与管理工作的指导意见》 [2]。在这个特殊时期, 各大慕课平台的视频资源全 部免费开放, 各高校积极响应, 利用现有资源, 依托各级各类在线学习平台, 开始线上建课, 为实 现 “停课不停教、停课不停学” 做好前期准备工作。但在 2 月 17 日全国大部分高校首日开学之际, 雨课堂、学习通等在线学习平台无法接纳使用量的瞬间增长, 为保证平台的正常运行, 各平台对平 台功能进行了调整，直播课限流。

在这种突发状况下, 为保证疫情防控期间的教学进度和教学质量, 很多教师开始使用一些直播 软件, 并结合原有在线学习平台展开教学工作。笔者以 “ $\mathrm{QQ}$ 分屏 + 学习通” 为例对化工原理课程 线上授课过程进行介绍。

收稿: 2020-02-26; 录用: 2020-04-19; 网络发表：2020-04-30

通讯作者, Email: mjjwjpmartin@163.com 


\section{1 前期准备}

\section{1 软件安装}

QQ 是一款大众即时通信软件, 在学生群体中有很高的使用率, 无需单独下载安装, 且 PC 端和 移动端可同时使用, 简单方便, 所以通过 QQ 建立班级群, 和学生沟通更及时更高效。超星学习通 是面向移动终端的一款教育 APP, 于 2019 年 12 月通过教育部备案 ${ }^{[3]}$, 需要在应用商城下载后使用, 支持安卓 5.0 或 IOS 8.0 及以上版本。学生使用学习通利用移动设备进行线上学习和课堂互动更简单 方便, 教师也可在 PC 端输入本校教学平台网址(如 http://hebau.fy.chaoxing.com)登录后和移动端具有 相同数据、功能, 在建课阶段从电脑端上传视频、文件、试题等资料更加便捷。

\section{2 软件简介}

学习通有 1000 多门课程的教学示范包, 内含教学课件、教学视频、试题库、作业库等多种资源。 课程中的活动库包括课堂活动、课程内容以及更多资料这三个模块。其中课堂活动较丰富, 包括签 到、投票、选人、抢答、主题讨论、测验、问卷、评分、分组任务、直播、学生反馈、通知、白板 等, 主要用于教师在线教学、课堂管理、疑难问题解答、师生互动环节。课程内容模块展示的是学 习资料、课后作业以及课程章节。更多资料部分主要是图片、视频、收藏、网页链接等。其中教学 示范包、课程内容以及更多资料模块主要用于课程建设。

\section{3 课程建设}

课程建设过程中, 教师可以在教学示范包中搜寻适合的课程直接引用, 一键建课。建课完成后 还可以结合本校学生情况自行录制视频, 并上传到更多资料模块对讲授内容进行补充调整。如果课 程教学团队有丰富的教学资源, 也可以自行建课, 在课程内容模块上传自制的视频、试题、课件等 学习资料。自制视频为了便于上传和学生观看时的稳定性, 建议将知识点分成若干个小模块, 每个 模块的视频以 10-15 min 为宜, 以 MP4 格式为佳。自建题库尽量以填空、判断、选择等客观题型为 主, 学习通系统可以自动批阅。也可适当准备一些主观题, 采取教师批阅、学生互评或两者结合的 评阅方式。

\section{4 导入学生}

学习通导入学生主要有两种模式, 一种是由学生扫码进班, 一种是由教师导入进班。如果教师 手里没有学生的选课名单, 可以选择由学生扫码进班, 教师在建班后, 会自动生成一个二维码和一 串数字(邀请码), 学生通过学习通首页右上角的 “邀请码” 输入邀请码或扫码二维码进班即可。如 果教师手里有学生的选课名单, 可以选择从电脑端批量导入学生, 这样的最大好处是期末导出平台 成绩时学生名单与选课名单一致, 便于成绩整理。

QQ 建群后可以建议学生将群昵称修改为 “专业班级 + 学号后两位 + 姓名” 的格式, 这样 QQ 群里的学生名单会按专业班级和学号进行排序, 在分享屏幕进行直播时可以很轻松地确定学生。

\section{2 线上授课模式}

\section{1 课前}

教师要提前做好每次线上上课的教学计划, 包括本次课的教学内容和教学方式, 最重要的是本 次课的教学流程, 如表 1 所示。

教学流程表可以提前发给学生, 让学生对线上课程的流程有一个大致的了解, 提前做好准备而 不至于手忙脚乱。

\section{2 课中}

学习通虽然课堂活动丰富, 但是在使用量高峰时段不能保证直播功能的正常使用。QQ 群中的 分享屏幕功能可以把教师端的电脑屏幕实时分享给学生, 进行录屏直播, 两者结合相得益彰。

\subsection{1 在线授课}

QQ 分屏与学习通的结合方式也多种多样, 教师可以利用学习通讲授, 通过 QQ 分享屏幕进行 直播授课。当网络拥堵直播无法使用时, 教师可以如表 1 所示, 利用学习通发布视频的定时功能, 
要求学生在指定时间内完成指定视频的观看任务, 然后通过测验了解学生对视频所讲知识点的理解 深度和广度, 并根据测验中所反映的问题调整后面录屏直播时的教授重点。教师也可以在电脑端播 放任务点视频, 通过 $\mathrm{QQ}$ 分享屏幕, 和学生一起观看视频, 对视频中晦涩难懂的地方可以直接暂停 进行讲解。结合学科特点、学生情况、平台功能, 教师可以有针对性地灵活应用。整个线上教学过 程中, 教师可以借助活动区的签到功能记录学生的学习痕迹, 并能量化计分, 便于课堂学习管理。

表 1 8:00-9:40 化工原理课程授课情况(以实际上课为准, 可能会有 1-2 $\mathrm{min}$ 的误差)

\begin{tabular}{|c|c|c|c|}
\hline 时间安排 & 任务 & 要求 & 备注 \\
\hline $7: 50-8: 00$ & 学习通签到 & 完成签到后的学生请随时关注学习通里的通知和消息 & 利用学习通完成 \\
\hline \multirow[t]{2}{*}{$8: 00-8: 10$} & 观看第一个视频 & 认真观看视频, 记录重要名词的意义、各种概念之间 & \\
\hline & 1.4 .1 精馏过程原理 & 的关系, 掌握气相、液相组成的变化 & \\
\hline $8: 10-8: 15$ & 完成测验 1.4 .1 & 仔细审题, 注意题干的叙述 & \\
\hline \multirow[t]{3}{*}{$8: 15-8: 20$} & 主题讨论发言 & 举例分析精馏操作时气相、液相中轻组分含量的变化 & \\
\hline & 精馏操作时气相、液相中轻组分 & & \\
\hline & 含量如何变化? & & \\
\hline \multirow[t]{2}{*}{$8: 20-8: 35$} & 观看第二个视频 & 认真观看视频, 记录重要名词的意义, 掌握精馏塔的 & \\
\hline & 1.4 .2 精馏塔模型 & 结构, 明确气相、液相组成的变化 & \\
\hline $8: 35-8: 45$ & 完成测验 1.4 .2 & 仔细审题, 注意题干的叙述 & \\
\hline \multirow[t]{2}{*}{$8: 45-8: 55$} & 课间休息 & 未完成两个测验的同学可在课间休息补交测验, 完成 & 利用 $\mathrm{QQ}$ 分享屏幕 \\
\hline & 重新发布测验 1.4 .1 和 1.4 .2 & 的同学休息 $10 \mathrm{~min}$ & 完成 \\
\hline \multirow[t]{3}{*}{$8: 52-8: 55$} & 进入 $\mathrm{QQ}$ 分享屏幕 & 进入的同学请自动关闭自己的麦克风, 我会在屏幕上 & \\
\hline & & 显示待进入学生名单, 各班班长和学委根据名单通知 & \\
\hline & & 学生进入, 我们 8:55 正式上课 & \\
\hline \multirow[t]{4}{*}{$8: 55-9: 25$} & 观看 QQ 分享屏幕 & 通过屏幕分享串讲前面的视频的内容, 讲解两次测验 & \\
\hline & & 错误率较高的题目, 补充部分典型例题, 期间可能会 & \\
\hline & & 随机选择若干同学进行连麦回答问题, 请同学们做好 & \\
\hline & & 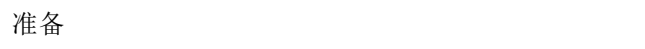 & \\
\hline \multirow[t]{2}{*}{$9: 25-9: 30$} & 完成测验 1.4 .3 的练习题 & 同学们在手机端用学习通完成 & $\mathrm{QQ}$ 分屏不结束, 学 \\
\hline & & & 生用学习通完成 \\
\hline \multirow[t]{2}{*}{$9: 30-9: 40$} & 观看 QQ 分享屏幕 & 同学们在手机端用学习通完成 & 利用 $\mathrm{QQ}$ 分享屏幕 \\
\hline & & & 完成 \\
\hline
\end{tabular}

\subsection{2 强化重点}

视频观看完毕, 教师可以针对气相、液相中轻组分的变化情况这种重点、难点问题进行更深层 次的讲解。教师可以借助 $\mathrm{QQ}$ 分享屏幕对 $t-x-y$ 相图中的气相、液相组成变化进行详细分析。教师也 可以利用学习通中的白板画出 $t-x-y$ 相图录制简短讲解视频, 方便学生重复观看。教师也可以通过学 习通发起主题讨论, 让学生就精馏过程中气相、液相组成变化情况分享观点, 教师在电脑端打开讨 论区, 通过分享屏幕和学生一起看留言区, 并对学生留言进行点评。教师还可以通过学习通发起投 票, 借助于判断题、选择题的形式了解学生对难点知识点的掌握情况, 并根据得分率对共性问题进 行再次讲授。

\subsection{3 师生互动}

在线授课过程中, 教师可以在 $\mathrm{QQ}$ 讨论区看到学生提出的问题, 也可以通过学习通中的学生反 
馈了解学生的疑问, 还可以借助学习通中的调查问卷分析学生对疑难问题的掌握情况。例如, 学生 不明白为什么塔顶的轻组分的含量高? 针对学生提出的问题, 教师可以通过 QQ 分享屏幕对学生提 出的问题进行实时讲解; 也可以通过学习通录制视频, 借助白板使用画笔对精馏塔过程中轻组分的 变化进行详细分析; 当然, 充分发挥在线教学的优势, 教师还可以通过网页搜索相关视频, 利用 QQ 分享屏幕与学生一起观看, 并及时进行查漏补缺, 解决学生的疑难问题。

此外, 教师还可以通过 $\mathrm{QQ}$ 分屏实现与学生连麦, 在调动学生课堂积极性的同时弥补线上教学 无法面对面交流的不足。例如, 针对 “精馏塔温度如何变化? ” 这种晦涩难懂的问题, 学生比较难 理解。通过连麦让学生回答轻组分的沸点低还是重组分的沸点低? 然后问学生塔顶轻组分的含量高 还是塔釜轻组分的含量高? 通过环环相扣的问题引导学生说出精馏塔塔顶温度低、塔釜温度高。这 样通过连麦引导学生对精馏塔温度的变化情况有更深入的理解。

\section{3 课后}

学习通中的视频任务点有多种设置形式, 对于课上已经学过的视频可以设置成公开, 学生结合 自身情况进行适当回放，补充笔记或加深理解，也可适当布置作业来巩固知识。

学习通有强大的电子图书资源, 可以免费阅读报纸文章以及中外文献元数据, 可以全文阅读和 下载中国知网的所有期刊论文。教师可以指导学生查阅一些和课程相关的文章, 拓展知识的广度和 深度。

\section{3 使用技巧}

\section{1 班长信息很重要}

在正式线上授课之前, 需要教务部门或学生部门提供班长的联系方式, 如果没有学生选课信息, 可以通过班长获取班级同学的学号、姓名信息, 便于在学习通和 $\mathrm{QQ}$ 群导入学生信息, 在学习通和 QQ 群中将班长设置为助教和管理员, 可以帮助教师发布通知, 管理 QQ 群, 提高效率。

\section{2 提前试课有必要}

如果学生是第一次使用学习通, 可能对签到、作业、主题讨论、投票等课堂活动的使用不是很 熟悉, 尽量线上授课之前进行试课, 试课主要是让学生熟悉学习通的各种功能及使用方法, 避免开 课后出现问题，耽误上课时间。

\section{3 注重细节提高在线上课质量}

(1) 在 QQ 分享屏幕时, 考虑到网络堵塞以及学生手机流量不足等问题, 尽量不要开启摄像头, 只用语音和屏幕画面进行直播。

(2) 给学生观看视频的时间要比视频的时长多 5-6 min, 要充分考虑到不是每个学生的网络状况 都很好, 要给出网络卡顿、加载视频的预留时间。

(3) 大部分学生放假回家, 没有准备教材, 课程组教师如有能力可以给学生提供所需章节教材 的扫描件, 或教学课件等资源。

(4) 教师尽量制订多种预案, 并用多种形式提供资源和开展教学, 确保教学的正常进行 ${ }^{[4]}$ 。

\section{参 考 文 献}

[1］２019 新型冠状病毒. [2020-02-13]. https://baike.so.com/doc/29084083-30563443.html.

[2] 教育部应对新型冠状病毒感染肺炎疫情工作领导小组办公室关于在疫情防控期间做好普通高等学校在线教学组织与管理工作的指导意 见. [2020-02-04]. http://www.moe.gov.cn/srcsite/A08/s7056/202002/t20200205_418138.html.

[3] 超星学习通百度百科. [2020-02-06].

https://baike.baidu.com/item/\%E8\%B6\%85\%E6\%98\%9F\%E5\%AD\%A6\%E4\%B9\%A0\%E9\%80\%9A/24248742.

[4] 王辉, 宋修艳. 大学化学, 2020,35(5), 3. 